\title{
Análisis del espacio euromediterráneo a través de las televisiones de la región: estudio de los informativos de ocho operadores públicos
}

\author{
Fernando SABÉS TURMO \\ Universitat Autònoma de Barcelona \\ fernando.sabes@uab.es \\ Ricardo CARNIEL BugS \\ Universitat Autònoma de Barcelona \\ ricardo.carniel@uab.es
}

Recibido: 11de diciembre de 2013

Aceptado: 14 de febrero de 2014

\begin{abstract}
Resumen
La información generada en el espacio euromediterráneo es cada vez de mayor importancia y no solamente por lo que se ha conocido como Primavera Árabe sino también por el esfuerzo realizado previamente desde diferentes foros como Proceso de Barcelona-Unión por el Mediterráneo, la Política Europea de Vecindad y las recomendaciones del Informe del Grupo de Alto Nivel de la Alianza de Civilizaciones. El artículo que presentamos aborda los enfoques y temas de las informaciones que ofrecieron los principales telenoticias de ocho canales públicos de televisión: ENTV (Argelia), ERTU (Egipto), TVE 1 (España), France 2 (Francia), RAI 1 (Italia), SNRT Al Aoula (Marruecos), BBC One (Reino Unido), Syria Channel (Siria). En total, se han estudiado 371 unidades de análisis recogidas entre el 9 y el 22 de marzo de 2009.
\end{abstract}

Palabras clave: Televisión, euromediterráneo, enfoque, temas, análisis de contenido

\section{Analysis of the Euro-Mediterranean area through the media of the region: the study of the newscasts of eight public TV broadcasters}

\begin{abstract}
The information generated in the Euro-Mediterranean area is increasingly important, not only for what has been known as Arab Spring but also for the effort previously made by different forums, such as the Barcelona Process - Union for the Mediterranean, the European Neighbourhood Policy and the recommendations of the Report of the High Level Group of the Alliance of Civilizations. This article analyses the focus and subjects of the information offered by the main newscast of eight public TV channels: ENTV (Algeria), ERTU (Egypt), France 2 (France), BBC One (UK), RAI 1 (Italy), SNRT Al Aoula (Morocco), Syria Channel (Syria), TVE 1 (Spain). The research considered 371 units of analysis that were collected between 9 and 22 March 2009.
\end{abstract}

Keywords: Television, Euro-Mediterranean, focus, subjects, content analysis

\section{Referencia normalizada}

SABÉS TURMO, Fernando y CARNIEL BLUGS, Ricardo (2014): "Análisis del espacio euromediterráneo a través de las televisiones de la región: estudio de los informativos de ocho operadores públicos". Estudios sobre el Mensaje Periodístico. Vol. 20, Núm. 2 (julio-diciembre), págs.: 885-897. Madrid, Servicio de Publicaciones de la Universidad Complutense.

Sumario: 1. Introducción. 2. Metodología, muestra y temas; 2.1. Metodología; 2.2. Muestra; 2.3. Enfoque y temas de las noticias. 3. Resultados del análisis. 4. Conclusiones. 5. Referencias bibliográficas.

\section{Introducción}

El estudio de lo que acontece bajo la perspectiva de la comunicación en el espacio euromediterráneo está siendo analizado desde hace varios años en el Laboratori de 
Prospectiva i Recerca en Comunicació, Cultura i Cooperació (LAPREC)' de la Universidad Autónoma de Barcelona a través de diferentes proyectos de investigación.

Este interés del LAPREC viene de lejos y se ha acrecentado con los acontecimientos que se han denominado como Primavera Árabe y sus consecuencias posteriores que todavía hoy vivimos y que muy probablemente las podremos seguir en los próximos años con cambios en la sociedad en este caso del sur del Mediterráneo. El norte, por su parte, no se mantiene impasible a lo que allí sucede como hemos podido comprobar durante las revueltas sociales y en sus derivadas siguientes.

El análisis de los medios de comunicación y sobre todo el estudio de la información que ofrecen es un elemento esencial para entender la agenda informativa en la cuenca mediterránea. Esta investigación debe enmarcarse dentro de las línea propuestas por el Proceso de Barcelona-Unión por el Mediterráneo, la Política Europea de Vecindad de la Comisión Europea y la Alianza de Civilizaciones adoptada por Naciones Unidas, esto es, acciones desarrolladas desde antes de las revueltas en países árabes en las que se trabaja por la integración regional.

Este artículo plantea el análisis de los principales enfoques y temas relativos al espacio euromediterráneo que aparecen en los telediarios de referencia de televisiones públicas que operan en países de la región, con el objetivo de verificar cómo se incorporan dichos asuntos en la agenda temática de estos medios y comprobar la relevancia o no del área mediterránea en los contenidos emitidos. Los principales elementos analizados que se detallarán posteriormente surgen de las líneas y acciones prioritarias destacadas por los principales documentos de las iniciativas anteriormente citadas: el Proceso de Barcelona-Unión por el Mediterráneo², la Política Europea de Vecindad de la Comisión Europea ${ }^{3}$ y las recomendaciones del Informe del Grupo de Alto Nivel de la Alianza de Civilizaciones contenidas en el Plan deAcción 2007-20094.

Los resultados que se apuntan en este texto son parte del resultado del proyecto de investigación dirigido por la Dra. Teresa Velázquez García-Talavera, titulado "La construcción social del espacio euromediterráneo en los medios de comunicación. La información en prensa y televisión"s.

1 Véase: http://grupsderecerca.uab.cat/laprec

2 Para más información se puede consultar: http://www.gencat.cat/relacions_exteriors /bcn10/cas/pdf/declaracio.pdf y http://www.ufmsecretariat.org/en/wp-content/uploads/2010 /11/ufm_paris_declaration1.pdf

${ }_{3}$ Para más información se puede consultar: http://ec.europa.eu/world/enp/pdf/country /enpi_euromed_rsp_summary_es.pdf

${ }^{4}$ Para más información se puede consultar: http://www.unaoc.org/docs/AoC_HLG_REPORT_SP.pdf y http://www.unaoc.org/wp-content/uploads/071010-Implementation-PיPlan1_2007-2009.pdf

5 "La construcción social del espacio euromediterráneo en los medios de comunicación. La información en prensa y televisión" (CSO2008-01579), financiada por el Ministerio español de Ciencia e Innovación (2009-2011). Investigadores por orden alfabético: Hassan Abbas, María Luz Barbeito, Tayeb Boutbouqalt, Ricardo Carniel Bugs, Carmina Crusafon, Olga Del Río, María Pilar Diezhandino Nieto, Natalia Fernández Díaz, Elisabet García Altadill, Juan Antonio García Galindo, Abdelouhad El Imrani, Francisco Martín, Carmen Martínez 
Esta investigación tiene su continuidad en la actualidad con un nuevo proyecto denominado "La construcción social del espacio intermediterráneo y sus correlaciones en la agenda temática de los medios de comunicación. La información en prensa y televisión", también financiado a través del Plan Nacional de investigación (CSO2012$35955)^{6}$.

\section{Metodología, muestra y temas}

\subsection{Metodología}

Este artículo adopta la metodología aplicada en el proyecto de investigación anteriormente citado. Esencialmente es de corte documental haciendo inicialmente un estudio previo a través de estadísticas y cifras de audiencia de los medios de comunicación, con el fin de obtener datos de las principales televisiones que actúan en el espacio euromediterráneo. De todas maneras, hay que indicar que en este proyecto también se hizo previamente un análisis de las principales cabeceras de prensa escrita que existen en la región. En total se estudiaron 16 diarios durante dos semanas. Sin embargo, este texto no hace referencia a los resultados obtenidos de la prensa escrita.

La metodología planteada parte de la teoría de la realidad social, es decir, cómo los medios de comunicación participan en esa construcción, considerando el papel relevante que tienen en la sociedad. Tal y como señalan Berger y Luckmann (1976), el contexto social condiciona la realidad y el conocimiento establecido. De hecho, podríamos hablar de una tendencia fenomenológica-interpretativa desde la perspectiva teórica. "Suele haber consenso en torno a la consideración de que la Sociología Fenomenológica se inserta en el campo de las llamadas Sociologías Interpretativas, junto a corrientes como el Interaccionismo Simbólico, las Sociologías de la Vida Cotidiana e incluso las Sociologías de la Situación" (Rizo, 2009: 25).

Por su parte, Velázquez (1992: 22) considera que "si lo medios de comunicación de masas contribuyen a la construcción de la realidad y la presentan en el espacio de la comunicación pública, los destinatarios de ese discurso también perciben y construyen realidad, bien la presentada por los medios, bien la individual según su conocimiento del mundo, valores, creencias, opiniones...".

Romero, Lucía Molina, Nieves Ortega, José María Perceval, José Manuel Pérez Tornero, Fernando Sabés, Susana Tovías, Sahar Taalat. Teresa Velázquez (Investigadora Principal). Doctorandas adscritas al proyecto: Cristina Marques y Valentina Saini.

6 "La construcción social del espacio intermediterráneo y sus correlaciones en la agenda temática de los medios de comunicación. La información en prensa y televisión" (CSO201235955) es un proyecto financiado por el Ministerio español de Economía y Competitividad (2013-2015). Investigadores por orden alfabético: Hesham Abu-Sharar, María Luz Barbeito, Ricardo Carniel Bugs, Carmina Crusafon, Olga Del Río, Lucrecia Escudero Chauvel, Natalia Fernández Díaz, Elisabet García Altadill, Juan Antonio García Galindo, Víctor Luís Gutiérrez Castillo, Nouredinne Harrami, Carmen Martínez Romero, Patria Román-Velázquez, Fernando Sabés, Susana Tovías, José Juan Verón Lassa y Teresa Velázquez (Investigadora Principal). Doctorandos adscritos al proyecto: Hicham El Bakhouti, Estibaliz Ortega Miranda y Valentina Saini. Investigadores colaboradores (pendientes de incorporación administrativa): Zhou Bouzidi y Carolina Cerdá-Guzmán. 
Bajo estas premisas se procedió a la selección de las televisiones que se incluirían en la muestra para la realización posterior de un análisis de contenido de sus principales informativos durante dos semanas.

En este estudio de contenido se consideró únicamente necesario abordar los temas y enfoques que hicieran referencia a la construcción social del espacio euromediterráneo. Para desarrollar esta tarea, se diseñó una ficha de análisis ampliamente discutida en el grupo de investigación y que fuera útil para radiografiar todas las televisiones seleccionadas de la región. Para comprobar su validez, se realizó un pretest que evidenció que era la adecuada para afrontar el trabajo que se pretendía llevar a cabo.

La ficha era eminentemente de corte cuantitativo, pero se incluyó también una parte cualitativa para desarrollar un análisis semiótico que también se aplicó a la muestra seleccionada. El objetivo era conocer "cómo se dice lo que se dice" para abordar el contenido latente del mensaje y su significado, de manera que se encuentren las claves para su interpretación (Velázquez, 2011: 237).

Sin embargo, en este artículo nos centraremos en la primera parte del análisis, el estudio cuantitativo, haciendo referencia a la agenda temática, es decir, que contenidos y enfoques vinculados con el espacio euromediterráneo incluyen las televisiones seleccionadas en sus telediarios. Este hecho nos permitirá conocer los principales temas que ofrecen estos medios de comunicación.

Por tanto, ofrecemos una parte de los resultados de este análisis de contenido cuantitativo. Tomaremos como referencia la definición ofrecida por Holsti (1968) quien señala que el análisis de contenido es un procedimiento que permite analizar y cuantificar los materiales de la comunicación; puede analizarse con detalle y profundidad el contenido de cualquier comunicación (código lingüístico oral, icónico, gestual, gestual signado...) sea cual fuere el número de personas implicadas en la comunicación (una, diálogo, grupo restringido, comunicación de masas...), pudiendo emplear cualquier instrumento de compendio de datos (agendas, diarios, cartas, cuestionarios, encuestas, tests proyectivos, libros, anuncios, entrevistas, radio, televisión, etc.).

Así, Bardin (1986) asegura que "el análisis de contenido es un conjunto de técnicas de análisis de comunicaciones que tiende a obtener indicadores (cuantitativos o no) por procedimientos sistemáticos y objetivos de descripción del contenido de los mensajes, permitiendo la inferencia de conocimientos relativos a las condiciones de producción/recepción (variables inferidas) de estos mensajes" (Bardin, 1986: 32).

"Esto ocurre por el descubrimiento de los contenidos y de las estructuras que confirman (o no) lo que se quiere demostrar a propósito de los mensajes, o por el esclarecimiento de los elementos significativos susceptibles de conducir a una descripción de mecanismos de los que a priori no detenemos la comprensión" (Bardin, 1995: 29).

Por su parte, Krippendorff (1990: 28) define el análisis de contenido como "la técnica destinada a formular, a partir de ciertos datos, inferencias reproducibles y válidas que puedan aplicarse a un contexto".

En consecuencia, el enfoque aquí planteado se inserta tanto dentro del Content Analysis de Estados Unidos, entendido, según Berelson, como "una técnica de investigación para la descripción objetiva, sistemática y cuantitativa del contenido manifiesto de las comunicaciones" (1986: 13), como en la tradición europea de estudios de contenido que toma como elementos de análisis la presentación de los mensajes, 
la valoración, emplazamiento, titulación y compaginación como categorías de medición, los elementos estructurales, la morfología del medio y las unidades redaccionales (Krippendorff, 1990).

En definitiva, el objetivo que nos marcamos con este trabajo es conocer la agenda y el enfoque informativo de las principales televisiones del ámbito euromediterráneo en relación a los temas que afectan directamente a la región de acuerdo a los planteamientos expresados por los principales documentos del Proceso de Barcelona-Unión por el Mediterráneo, la Política Europea de Vecindad de la Comisión Europea y las recomendaciones del Informe del Grupo de Alto Nivel de la Alianza de Civilizaciones contenidas en el Plan de Acción 2007-2009.

\subsection{Muestra}

La muestra del estudio corresponde a los informativos diarios en prime time de ocho de las principales televisiones públicas del ámbito euromediterráneo. La muestra se recogió durante dos semanas, del 9 al 22 de marzo de 2009. Las unidades de análisis resultantes fueron 371 , correspondientes 216 a la primera semana y 155 a la segunda, es decir, un $58,2 \%$ frente a un $41,8 \%$. Los países escogidos y sus respectivos canales públicos fueron: Argelia (ENTV), Egipto (ERTU), España (TVE 1), Francia (France 2), Italia (RAI 1), Marruecos (SNRT Al Aoula), Reino Unido (BBC One) y Siria (Syria Channel).

De los canales estudiados, cuatro emiten en árabe (ENTV, ERTU, SNRT Al Aoula y Syria Channel), una en inglés (BBC One), una en francés (France 2), una en italiano (RAI 1) y una en español (TVE 1).

En cuanto a la participación de las emisoras en la muestra, ésta se reparte de la forma siguiente: ERTU (25,6\%, 95 piezas), Syria Channel $(18,6 \%, 69)$, RAI 1 (15,6\%, $58)$, ENTV $(12,9 \%, 48)$, TVE $1(9,7 \%, 36)$, SNRT Al Aoula $(9,7 \%$, 36), France 2 $(5,1 \%, 19)$ y BBC One $(2,7 \%, 10)$.

Por tanto, el idioma mayoritario utilizado en las unidades de análisis incluidas en la muestra fue el árabe $(66,9 \%)$. Por otra parte, la proporción de noticias de los países europeos corresponde al número de noticias de cada canal: italiano $(15,6 \%)$, español $(9,7 \%)$, francés $(5,1 \%)$ e inglés $(2,7 \%)$.

\subsection{Enfoques y temas de las noticias}

Para analizar las noticias relacionadas con el espacio euromediterráneo fue diseñada una ficha de análisis con más de 70 variables, entre aspectos formales o técnicos (utilización de imágenes, conexiones en directo, duración de la noticia, etc.), la jerarquización de las noticias (sumario de entrada, orden de la escaleta, etc.) y los elementos relacionados concretamente con la información difundida. En el presente artículo, ponemos el foco sobre este último conjunto de variables, el tratamiento de las noticias, especialmente sobre el enfoque principal y los temas incluidos en cada una de las unidades de análisis.

Al contrario del análisis de la prensa, en la que se puede identificar de forma explícita las secciones en las cuales cada periódico organiza sus contenidos, los informativos de televisión no suelen utilizar una división tan evidente. En este sentido, además de tener en cuenta la jerarquización de las noticias en la escaleta de los tele- 
noticias, fue incluida la variable de "enfoque principal de la noticia", con el fin de observar la relación entre los temas abordados y el tratamiento periodístico de las noticias. Así, fueron definidos 11 enfoques principales: "Comunicación/Medios", "Cultural", "Deportivo", "Económico", "Judicial", "Militar/Bélico", "Policial", "Político", "Religioso", "Sucesos/Catástrofes" y "Social".

Con relación a los temas presentes en las noticias, partimos de los principales documentos de las tres iniciativas anteriormente mencionadas: las declaraciones fundacionales del Proceso de Barcelona-Unión por el Mediterráneo, la Política Europea de Vecindad de la Comisión Europea y las recomendaciones del Informe del Grupo de Alto Nivel de la Alianza de Civilizaciones contenidas en el Plan de Acción 2007-2009. A partir de dichos documentos se verificó la existencia de valores, principios y, sobre todo, ejes de acción prioritarios que coincidían en las tres instancias, así como el objetivo central de promover la construcción de un espacio euromediterráneo. Por tanto, de estos ejes principales se han extraído los temas que se buscaban verificar en las noticias emitidas por las televisiones públicas mediterráneas. Del conjunto de temas encontrados, en este artículo nos ceñiremos a los temas de "Política y Legislación", "Economía y finanzas", "Cultura" y "Religión", todos ellos considerados de extrema relevancia por las políticas de integración regional.

\section{Resultados del análisis}

Del total de las noticias analizadas, el 53,6\% tienen como principal enfoque el político. Le sigue el económico (15,4\%), el social $(10,8 \%)$, el militar/bélico (7,3\%), el religioso $(3,2 \%)$, el cultural $(3,2 \%)$, el policial $(3 \%)$, el judicial $(2,4 \%)$, los sucesos/catástrofes $(0,5 \%)$, la comunicación/medios $(0,3 \%)$ y el deportivo $(0,3 \%)$.

Sí hay que indicar que el peso de cada una de las cadenas de televisión sobre la muestra incluida cambia de forma sustancial. De hecho, la emisora que aporta porcentualmente más casos de estudio es el ente público ERTU, con el 25,6\%. Le siguen Syria Channel $(18,6 \%)$, RAI $1(15,6 \%)$ y ENTV $(12,9 \%)$. Por su parte, TVE 1 y SNRT Al Aoula suman cada una de ellas el $9,7 \%$ de la muestra. Cierran con menor repercusión de las noticias vinculadas al espacio euromediterráneo France 2, con 5,1\%, y BBC One, con 2,7\%.

En la cadena egipcia ERTU, el enfoque principal hace referencia contenidos políticos, mientras que el resto tienen una escasa repercusión en sus informativos: económico, social y militar/bélico.

En Syria Channel de nuevo el enfoque político es el que predomina, aunque completan la oferta informativa los enfoques militar/bélico, seguido del económico, social y religioso.

En la italiana RAI 1, los enfoques político, económico y social tienen un peso muy similar, aunque también encontramos otros como el policial, judicial, religioso, sucesos/catástrofes y de forma mínima el cultural, la comunicación/medios y militar/bélico.

En ENTV de Argelia, el enfoque político predomina mayoritariamente, mientras que el resto de enfoques tienen una repercusión mínima: económico, cultural, religioso, social y militar/bélico.

El canal público español TVE 1 incluye como enfoques mayoritarios el económico y el social, seguidos del político, militar/bélico, judicial, cultural y policial. 
En la emisora marroquí SNRT Al Aoula su principal enfoque es el político, seguido del económico, religioso, cultural, social, policial, deportivo y militar/bélico, estos tres últimos con un mismo peso.

France 2 incluye el enfoque social como el más relevante, seguido de político, económico, militar/bélico, religioso, policial y cultural.

En la británica, BBC One el enfoque más representativo es el social, seguido de económico, cultural y judicial.

\begin{tabular}{|c|c|c|c|c|c|c|c|c|c|c|c|c|}
\hline \multirow[b]{2}{*}{ TV } & \multicolumn{11}{|c|}{ Tabla 1. Enfoque principal de la noticia } & \multirow[b]{2}{*}{ Total } \\
\hline & $\begin{array}{c}\text { Comunicación } \\
\text { Medios }\end{array}$ & Cultural & Deportivo & Económico & Judicial & $\begin{array}{c}\text { Militar } \\
\text { I } \\
\text { Bélico }\end{array}$ & Policial & Político & Religioso & Social & $\begin{array}{c}\text { Sucesos } \\
\underset{1}{\text { Catástrofes }}\end{array}$ & \\
\hline \multirow{2}{*}{$\begin{array}{l}\text { BBC One } \\
\text { (Reino } \\
\text { Unido) }\end{array}$} & 0 & 2 & 0 & 3 & 1 & 0 & 0 & 0 & 0 & 4 & 0 & 10 \\
\hline &, $0 \%$ &, $5 \%$ &, $0 \%$ &, $8 \%$ &, $3 \%$ &, $0 \%$ &, $0 \%$ &, $0 \%$ &, $0 \%$ & $1,1 \%$ &, $0 \%$ & $2,7 \%$ \\
\hline \multirow{2}{*}{$\begin{array}{l}\text { ENTV } \\
\text { (Argelia) }\end{array}$} & 0 & 2 & 0 & 3 & 0 & 1 & 0 & 40 & 1 & 1 & 0 & 48 \\
\hline &, $0 \%$ &, $5 \%$ &, $0 \%$ &, $8 \%$ &, $0 \%$ &, $3 \%$ &, $0 \%$ & $10,8 \%$ &, $3 \%$ & $3 \%$ &, $0 \%$ & $12,9 \%$ \\
\hline \multirow{2}{*}{$\begin{array}{l}\text { ERTU } \\
\text { (Egipto) }\end{array}$} & 0 & 0 & 0 & 10 & 0 & 1 & 0 & 82 & 0 & 2 & 0 & 95 \\
\hline &, $0 \%$ &, $0 \%$ &, $0 \%$ & $2,7 \%$ &, $0 \%$ &, $3 \%$ &, $0 \%$ & $22,1 \%$ &, $0 \%$ &, $5 \%$ &, $0 \%$ & $25,6 \%$ \\
\hline \multirow{2}{*}{$\begin{array}{l}\text { France } 2 \\
\text { (Francia) }\end{array}$} & 0 & 1 & 0 & 3 & 0 & 3 & 1 & 4 & 2 & 5 & 0 & 19 \\
\hline &, $0 \%$ &, $3 \%$ &, $0 \%$ &, $8 \%$ &, $0 \%$ &, $8 \%$ &, $3 \%$ & $1,1 \%$ &, $5 \%$ & $1,3 \%$ &, $0 \%$ & $5,1 \%$ \\
\hline \multirow{2}{*}{$\begin{array}{l}\text { RAI 1 } \\
\text { (Italia) }\end{array}$} & 1 & 1 & 0 & 13 & 5 & 1 & 7 & 14 & 2 & 12 & 2 & 58 \\
\hline &, $3 \%$ &, $3 \%$ &, $0 \%$ & $3,5 \%$ & $1,3 \%$ &, $3 \%$ & $1,9 \%$ & $3,8 \%$ &, $5 \%$ & $3,2 \%$ &, $5 \%$ & $15,6 \%$ \\
\hline \multirow{2}{*}{$\begin{array}{l}\text { SNRT } \\
\text { Al Aoula } \\
\text { (Marruecos) }\end{array}$} & 0 & 4 & 1 & 6 & 0 & 1 & 1 & 16 & 5 & 2 & 0 & 36 \\
\hline &, $0 \%$ & $1,1 \%$ &, $3 \%$ & $1,6 \%$ &, $0 \%$ &, $3 \%$ &, $3 \%$ & $4,3 \%$ & $1,3 \%$ &, $5 \%$ &, $0 \%$ & $9,7 \%$ \\
\hline \multirow{2}{*}{$\begin{array}{l}\text { Syria } \\
\text { Channel } \\
\text { (Siria) }\end{array}$} & 0 & 0 & 0 & 10 & 0 & 15 & 0 & 37 & 2 & 5 & 0 & 69 \\
\hline &, $0 \%$ &, $0 \%$ &, $0 \%$ & $2,7 \%$ &, $0 \%$ & $4,0 \%$ &, $0 \%$ & $10,0 \%$ &, $5 \%$ & $1,3 \%$ &, $0 \%$ & $18,6 \%$ \\
\hline \multirow{2}{*}{$\begin{array}{l}\text { TVE } 1 \\
\text { (España) }\end{array}$} & 0 & 2 & 0 & 9 & 3 & 5 & 2 & 6 & 0 & 9 & 0 & 36 \\
\hline &, $0 \%$ &, $5 \%$ &, $0 \%$ & $2,4 \%$ &, $8 \%$ & $1,3 \%$ &, $5 \%$ & $1,6 \%$ &, $0 \%$ & $2,4 \%$ &, $0 \%$ & $9,7 \%$ \\
\hline \multirow{2}{*}{ Total } & 1 & 12 & 1 & 57 & 9 & 27 & 11 & 199 & 12 & 40 & 2 & 371 \\
\hline &, $3 \%$ & $3,2 \%$ &, $3 \%$ & $15,4 \%$ & $2,4 \%$ & $7,3 \%$ & $3,0 \%$ & $53,6 \%$ & $3,2 \%$ & $10,8 \%$ &, $5 \%$ & $100,0 \%$ \\
\hline
\end{tabular}

Fuente: Elaboración propia

El enfoque mayoritario, el político, coincide con los temas más presentes en las noticias estudiadas, concernientes a la variable Política y Legislación. El tema más tratado es políticas multilaterales o bilaterales, con un $33,2 \%$, seguido de derecho internacional y derechos humanos $(15,6 \%)$, partidos políticos $(11,1 \%)$, liderazgo político responsable $(6,2 \%)$, integridad regional y/o autodeterminación de los pueblos $(3,8 \%)$ y democracia y buena gobernanza $(3,2 \%)$. También es pertinente observar el porcentaje de noticias que no hicieron referencia a ninguno de los valores (No hay), esto es, $26,1 \%$ de las noticias analizadas no trataron aspectos concernientes a política y legislación.

En cuanto al análisis por cadenas, las políticas multilaterales/bilaterales son el contenido más relevante en ERTU (8,9\% sobre el total de informaciones incluidas en la muestra). Le sigue partidos políticos $(7,3 \%)$, liderazgo político responsable $(3,5)$, derecho internacional y derechos humanos $(2,7 \%)$, democracia y buena gobernanza $(0,5 \%)$ e integridad regional y/o autodeterminación de los pueblos $(0,5 \%)$.

En Syria Channel el subtema con mayor repercusión es políticas multilaterales/bilaterales con $7,8 \%$, seguido de derecho internacional y derechos humanos $(6,5 \%)$, integridad regional y/o autodeterminación de los pueblos $(1,6 \%)$, partidos políticos $(1,1 \%)$ y liderazgo político responsable $(0,5 \%)$. 
En la italiana RAI 1, de nuevo, la políticas multilaterales/bilaterales representan el contenido mayoritario con 5,4\%. A continuación hallamos derecho internacional y derechos humanos $(1,6 \%)$, partidos políticos $(0,8 \%)$, democracia y buena gobernanza $(0,3 \%)$, liderazgo político responsable $(0,3 \%)$ e integridad regional y/o autodeterminación de los pueblos $(0,3 \%)$.

En ENTV, las políticas multilaterales/bilaterales representan el 4\%. Le sigue derecho internacional y derechos humanos $(2,2 \%)$, democracia y buena gobernanza $(1,6 \%)$, partidos políticos $(1,3 \%)$, liderazgo político responsable $(0,8 \%)$ e integridad regional y/o autodeterminación de los pueblos $(0,5 \%)$.

En TVE 1, las políticas multilaterales/bilaterales alcanzan el 1,9\%. Observamos a continuación el derecho internacional y derechos humanos $(1,3 \%)$, democracia y buena gobernanza $(0,5 \%)$ e integridad regional y/o autodeterminación de los pueblos $(0,3 \%)$.

SNRT 1 Al Aoula aporta sobre el global de la muestra el 4,9\% de informaciones vinculadas a políticas multilaterales/bilaterales. Le sigue liderazgo político responsable y derecho internacional y derechos humanos, ambos con un $0,8 \%$. Por últimos hallamos a partidos políticos, con un $0,5 \%$.

En France 2 únicamente localizamos tres temas: integridad regional y/o autodeterminación de los pueblos $(0,5 \%)$, derechos internacional y derechos humanos $(0,3 \%)$ y democracia y buena gobernanza $(0,3 \%)$.

La británica BBC One también incorpora tres temas, todos ellos con el mismo porcentaje, $0,3 \%$ : políticas multilaterales/bilaterales, liderazgo político responsable y derecho internacional y derechos humanos.

\begin{tabular}{|c|c|c|c|c|c|c|c|c|c|}
\hline \multirow[b]{2}{*}{ TV } & \multicolumn{8}{|c|}{ Tabla 2. Política y legislación } & \multirow[b]{2}{*}{ Total } \\
\hline & $\begin{array}{c}\text { Democracia y } \\
\text { buena } \\
\text { gobernanza }\end{array}$ & $\begin{array}{c}\text { Derecho } \\
\text { internacional y } \\
\text { derechos } \\
\text { humanos }\end{array}$ & $\begin{array}{l}\text { Integridad regional y/o } \\
\text { autodeterminación de } \\
\text { los pueblos }\end{array}$ & $\begin{array}{l}\text { Liderazgo } \\
\text { político } \\
\text { responsable }\end{array}$ & No hay & Otro & $\begin{array}{l}\text { Partidos } \\
\text { políticos }\end{array}$ & $\begin{array}{l}\text { Políticas } \\
\text { multilate- } \\
\text { rales / } \\
\text { bilaterales }\end{array}$ & \\
\hline \multirow{2}{*}{$\begin{array}{l}\text { BBC One } \\
\text { (Reino Unido) }\end{array}$} & 0 & 1 & 0 & 1 & 6 & 1 & 0 & 1 & 10 \\
\hline &, $0 \%$ &, $3 \%$ &, $0 \%$ &, $3 \%$ & $1,6 \%$ &, $3 \%$ &, $0 \%$ &, $3 \%$ & $2,7 \%$ \\
\hline \multirow{2}{*}{ ENTV (Argelia) } & 6 & 8 & 2 & 3 & 9 & 0 & 5 & 15 & 48 \\
\hline & $1,6 \%$ & $2,2 \%$ &, $5 \%$ &, $8 \%$ & $2,4 \%$ &, $0 \%$ & $1,3 \%$ & $4,0 \%$ & $12,9 \%$ \\
\hline \multirow{2}{*}{ ERTU (Egipto) } & 2 & 10 & 2 & 13 & 8 & 0 & 27 & 33 & 95 \\
\hline &, $5 \%$ & $2,7 \%$ &, $5 \%$ & $3,5 \%$ & $2,2 \%$ &, $0 \%$ & $7,3 \%$ & $8,9 \%$ & $25,6 \%$ \\
\hline \multirow{2}{*}{$\begin{array}{l}\text { France 2 } \\
\text { (Francia) }\end{array}$} & 1 & 1 & 2 & 0 & 15 & 0 & 0 & 0 & 19 \\
\hline &, $3 \%$ &, $3 \%$ &, $5 \%$ &, $0 \%$ & $4,0 \%$ &, $0 \%$ &, $0 \%$ &, $0 \%$ & $5,1 \%$ \\
\hline \multirow{2}{*}{$\begin{array}{l}\text { RAI 1 } \\
\text { (Italia) }\end{array}$} & 1 & 6 & 1 & 1 & 26 & 0 & 3 & 20 & 58 \\
\hline &, $3 \%$ & $1,6 \%$ & ,3\% &, $3 \%$ & $7,0 \%$ &, $0 \%$ &, $8 \%$ & $5,4 \%$ & $15,6 \%$ \\
\hline \multirow{2}{*}{$\begin{array}{l}\text { SNRT } \\
\text { Al Aoula } \\
\text { (Marruecos) }\end{array}$} & 0 & 3 & 0 & 3 & 10 & 0 & 2 & 18 & 36 \\
\hline &, $0 \%$ &, $8 \%$ &, $0 \%$ &, $8 \%$ & $2,7 \%$ &, $0 \%$ &, $5 \%$ & $4,9 \%$ & $9,7 \%$ \\
\hline \multirow{2}{*}{$\begin{array}{l}\text { Syria Channel } \\
\text { (Siria) }\end{array}$} & 0 & 24 & 6 & 2 & 2 & 2 & 4 & 29 & 69 \\
\hline &, $0 \%$ & $6,5 \%$ & $1,6 \%$ &, $5 \%$ &, $5 \%$ &, $5 \%$ & $1,1 \%$ & $7,8 \%$ & $18,6 \%$ \\
\hline \multirow{2}{*}{ TVE 1 (España) } & 2 & 5 & 1 & 0 & 21 & 0 & 0 & 7 & 36 \\
\hline &, $5 \%$ & $1,3 \%$ &, $3 \%$ &, $0 \%$ & $5,7 \%$ &, $0 \%$ &, $0 \%$ & $1,9 \%$ & $9,7 \%$ \\
\hline \multirow{2}{*}{ TOTAL } & 12 & 58 & 14 & 23 & 97 & 3 & 41 & 123 & 371 \\
\hline & $3,2 \%$ & $15,6 \%$ & $3,8 \%$ & $6,2 \%$ & $26,1 \%$ &, $8 \%$ & $11,1 \%$ & $33,2 \%$ & $100,0 \%$ \\
\hline
\end{tabular}

Fuente: Elaboración propia 
Aunque la economía es un asunto de referencia en el enfoque de las informaciones incluidas en la muestra sí hay que señalar que no está presente como tema principal en el 73,3\% de las piezas estudiadas.

La crisis económica mundial aparece en el 10\% del total de las informaciones incluidas en la muestra, es decir, en 37 piezas, seguida de recursos naturales y medioambiente $(2,7 \%)$, reformas financieras-fomento de inversiones $(2,4 \%)$, infraestructuras y transportes $(2,2 \%)$, integración económica Sur-Sur $(1,9 \%)$, pobreza y/o desigualdades económicas $(1,9 \%)$, recursos energéticos $(1,6 \%)$, zona euromediterránea de libre comercio $(1,6 \%)$, desarrollo sostenible $(1,3 \%)$ y cooperación empresarial/industrial $(0,8 \%)$.

La crisis económica mundial representa en ERTU el 2,4\% del total de las informaciones incluidas en la muestra. A continuación observamos el contenido integración económica Sur-Sur $(0,8 \%)$, al igual que los recursos energéticos $(0,8 \%)$. Completan los temas: zona euromediterránea de libre comercio $(0,5 \%)$, cooperación empresarial/industrial $(0,3 \%)$, infraestructuras y transportes $(0,3 \%)$, pobreza y/o desigualdades económicas $(0,3 \%)$, recursos naturales y medioambiente $(0,3 \%)$ y reformas financieras-fomento de inversiones $(0,3 \%)$.

En Syria Channel la economía aparece de forma más relevante a través de informaciones que hacen referencia a la crisis económica mundial $(1,1 \%)$. También hallamos recursos energéticos $(0,8 \%)$, infraestructuras y transportes $(0,5 \%)$, recursos naturales y medioambiente $(0,5 \%)$, reformas financieras-fomento de inversiones $(0,5 \%)$, desarrollo sostenible $(0,3 \%)$, integración económica Sur-Sur $(0,3 \%)$ y zona euromediterránea de libre comercio $(0,3 \%)$.

En RAI 1, la crisis económica mundial representa el 4\%. Le sigue pobreza y/o desigualdades económicas $(1,3 \%)$, infraestructuras y transportes $(0,5 \%)$, recursos naturales y medioambiente $(0,5 \%)$, cooperación empresarial/industrial $(0,3 \%)$ y reformas financieras-fomento de inversiones $(0,3 \%)$.

La argelina ENTV aporta el 0,5\% de las noticias analizadas bajo la temática desarrollo sostenible y el mismo porcentaje en infraestructuras y transportes y en recursos naturales y medioambiente. Completan este capítulo cooperación empresarial/industrial $(0,3 \%)$ e integración económica Sur-Sur $(0,3 \%)$.

TVE 1 recoge el 1,6\% de las informaciones a través de abordar la crisis económica mundial. Las reformas financieras-fomento de inversiones representan el 0,5\%, la integración económica Sur-Sur (3\%), pobreza y/o desigualdades económicas (3\%) y zona euromediteránea de libre comercio (3\%).

En SNRT Al Aoula observamos la presencia del contenido económico a través de cinco subtemas: desarrollo sostenible $(0,5 \%)$, recursos naturales y medioambiente $(0,5 \%)$, infraestructuras y transportes $(0,3 \%)$, integración económica Sur-Sur $(0,3 \%)$ y reformas financieras-fomento de inversiones $(0,3 \%)$.

En France 2 únicamente hay dos referencias al aspecto económico: reformas financieras-fomento de inversiones $(0,5 \%)$ y zona euromediterránea de libre comercio $(0,3 \%)$.

La británica BBC One incluye dos subtemas: crisis económica mundial $(0,8 \%)$ y recursos naturales y medioambiente $(0,3 \%)$. 


\begin{tabular}{|c|c|c|c|c|c|c|c|c|c|c|c|c|c|}
\hline \multirow[b]{2}{*}{ TV } & \multicolumn{12}{|c|}{ Tabla 3. Economía y finanzas } & \multirow[b]{2}{*}{ Total } \\
\hline & $\begin{array}{c}\text { Cooperación } \\
\text { empresarial/ } \\
\text { industrial }\end{array}$ & $\begin{array}{c}\text { Crisis } \\
\text { Económica } \\
\text { mundial }\end{array}$ & $\begin{array}{c}\text { Desarrollo } \\
\text { sostenible }\end{array}$ & $\begin{array}{c}\text { Infraestructuras } \\
\mathrm{y} \text { transportes }\end{array}$ & $\begin{array}{c}\text { Integración } \\
\text { económica } \\
\text { Sur - Sur }\end{array}$ & No hay & Otro & $\begin{array}{c}\text { Pobreza y/o } \\
\text { desigualdades } \\
\text { económicas }\end{array}$ & $\begin{array}{c}\text { Recursos } \\
\text { energéticos }\end{array}$ & \begin{tabular}{|c|} 
Recursos \\
naturales \\
y medio \\
ambiente
\end{tabular} & \begin{tabular}{|c|} 
Reformas \\
financieras, \\
fomento de \\
inversiones
\end{tabular} & \begin{tabular}{|c|} 
Zona euro- \\
Mediterránea \\
de libre \\
comercio
\end{tabular} & \\
\hline \multirow{2}{*}{$\begin{array}{l}\text { BBC One } \\
\text { (Reino } \\
\text { Unido) }\end{array}$} & 0 & 3 & 0 & 0 & 0 & 6 & 0 & 0 & 0 & 1 & 0 & 0 & 10 \\
\hline &, $0 \%$ &, $8 \%$ &, $0 \%$ &, $0 \%$ &, $0 \%$ & $1,6 \%$ &, $0 \%$ &, $0 \%$ &, $0 \%$ &, $3 \%$ &, $0 \%$ &, $0 \%$ & $2,7 \%$ \\
\hline \multirow{2}{*}{$\begin{array}{l}\text { ENTV } \\
\text { (Argelia) }\end{array}$} & 1 & 0 & 2 & 2 & 1 & 40 & 0 & 0 & 0 & 2 & 0 & 0 & 48 \\
\hline &, $3 \%$ &, $0 \%$ &, $5 \%$ &, $5 \%$ & $3 \%$ & $10,8 \%$ &, $0 \%$ &, $0 \%$ &, $0 \%$ &, $5 \%$ &, $0 \%$ &, $0 \%$ & $12,9 \%$ \\
\hline \multirow{2}{*}{$\begin{array}{l}\text { ERTU } \\
\text { (Egipto) }\end{array}$} & 1 & 9 & 0 & 1 & 3 & 73 & 0 & 1 & 3 & 1 & 1 & 2 & 95 \\
\hline &, $3 \%$ & $2,4 \%$ &, $0 \%$ &, $3 \%$ &, $8 \%$ & $19,7 \%$ &, $0 \%$ &, $3 \%$ &, $8 \%$ &, $3 \%$ &, $3 \%$ &, $5 \%$ & $25,6 \%$ \\
\hline \multirow{2}{*}{$\begin{array}{l}\text { France } 2 \\
\text { (Francia) }\end{array}$} & 0 & 0 & 0 & 0 & 0 & 16 & 0 & 0 & 0 & 0 & 2 & 1 & 19 \\
\hline &, $0 \%$ &, $0 \%$ &, $0 \%$ &, $0 \%$ &, $0 \%$ & $4,3 \%$ &, $0 \%$ &, $0 \%$ &, $0 \%$ &, $0 \%$ &, $5 \%$ &, $3 \%$ & $5,1 \%$ \\
\hline \multirow{2}{*}{$\begin{array}{l}\text { RAI } 1 \\
\text { (Italia) }\end{array}$} & 1 & 15 & 0 & 2 & 0 & 32 & 0 & 5 & 0 & 2 & 1 & 0 & 58 \\
\hline & $3 \%$ & $4,0 \%$ &, $0 \%$ &, $5 \%$ &, $0 \%$ & $8,6 \%$ &, $0 \%$ & $1,3 \%$ &, $0 \%$ &, $5 \%$ &, $3 \%$ &, $0 \%$ & $15,6 \%$ \\
\hline \multirow{2}{*}{$\begin{array}{l}\text { SNRT } \\
\text { Al Aoula } \\
\text { (Marruecos) }\end{array}$} & 0 & 0 & 2 & 1 & 1 & 27 & 1 & 0 & 0 & 2 & 1 & 1 & 36 \\
\hline &, $0 \%$ &, $0 \%$ &, $5 \%$ &, $3 \%$ &, $3 \%$ & $7,3 \%$ &, $3 \%$ &, $0 \%$ &, $0 \%$ &, $5 \%$ &, $3 \%$ &, $3 \%$ & $9,7 \%$ \\
\hline \multirow{2}{*}{$\begin{array}{l}\text { Syria } \\
\text { Channel } \\
\text { (Siria) }\end{array}$} & 0 & 4 & 1 & 2 & 1 & 53 & 0 & 0 & 3 & 2 & 2 & 1 & 69 \\
\hline &, $0 \%$ & $1,1 \%$ &, $3 \%$ &, $5 \%$ &, $3 \%$ & $14,3 \%$ & $0 \%$ &, $0 \%$ &, $8 \%$ &, $5 \%$ &, $5 \%$ &, $3 \%$ & $18,6 \%$ \\
\hline \multirow{2}{*}{$\begin{array}{l}\text { TVE 1 } \\
\text { (España) }\end{array}$} & 0 & 6 & 0 & 0 & 1 & 25 & 0 & 1 & 0 & 0 & 2 & 1 & 36 \\
\hline &, $0 \%$ & $1,6 \%$ &, $0 \%$ &, $0 \%$ &, $3 \%$ & $6,7 \%$ &, $0 \%$ &, $3 \%$ &, $0 \%$ &, $0 \%$ &, $5 \%$ &, $3 \%$ & $9,7 \%$ \\
\hline \multirow{2}{*}{ Total } & 3 & 37 & 5 & 8 & 7 & 272 & 1 & 7 & 6 & 10 & 9 & 6 & 371 \\
\hline &, $8 \%$ & $10,0 \%$ & $1,3 \%$ & $2,2 \%$ & $1,9 \%$ & $73,3 \%$ &, $3 \%$ & $1,9 \%$ & $1,6 \%$ & $2,7 \%$ & $2,4 \%$ & $1,6 \%$ & $100,0 \%$ \\
\hline
\end{tabular}

Fuente: Elaboración propia

Las referencias a la cultura en las informaciones analizadas en el estudio solamente suman el 8,6\% distribuidas de la siguiente forma: patrimonio cultural $(3,2 \%)$, diálogo y conocimiento mutuo $(2,2, \%)$, relaciones interculturales $(1,6 \%)$, identidad cultural $(1,1 \%)$ y especificidades culturales $(0,5 \%)$. Los otros $91,4 \%$ de las noticias consideradas no trataron ningún tema relacionado con la cultura.

ERTU únicamente incluye la temática patrimonio cultural que representa el 0,5\% del total de las informaciones analizadas en la muestra.

En Syria Channel hallamos las relaciones interculturales $(0,5 \%)$, especificidades culturales $(0,3 \%)$ e identidad cultural $(0,3 \%)$.

Dos temas encontramos en la televisión italiana RAI 1: diálogo y conocimiento mutuo $(0,5 \%)$ y patrimonio cultural $(0,3 \%)$.

La argelina ENTV recoge los siguientes contenidos culturales: relaciones interculturales $(0,8 \%)$, identidad cultural $(0,5 \%)$, especificidades culturales $(0,3 \%)$ y patrimonio cultural $(0,3 \%)$.

En TVE 1 observamos la presencia de diálogo y conocimiento mutuo $(0,5 \%)$ y patrimonio cultural $(0,5 \%)$.

En SNRT Al Aoula observamos como ofrece en el apartado cultura tres tipologías de informaciones: diálogo y conocimiento mutuo $(1,1 \%)$, patrimonio cultural $(1,1 \%)$ e identidad cultural $(0,3 \%)$.

En BBC One, el patrimonio cultural representa el $0,5 \%$ de las informaciones de la muestra y relaciones interculturales el 0,3\%.

\begin{tabular}{|c|c|c|c|c|c|c|c|}
\hline \multirow[b]{2}{*}{ TV } & \multicolumn{6}{|c|}{ Tabla 4. Cultura } & \multirow[b]{2}{*}{ Total } \\
\hline & $\begin{array}{c}\text { Diálogo y } \\
\text { conocimiento mutuo }\end{array}$ & $\begin{array}{l}\text { Especificidades } \\
\text { culturales }\end{array}$ & Identidad cultural & No hay & $\begin{array}{l}\text { Patrimonio } \\
\text { cultural }\end{array}$ & $\begin{array}{c}\text { Relaciones } \\
\text { interculturales }\end{array}$ & \\
\hline \multirow{2}{*}{$\begin{array}{l}\text { BBC One } \\
\text { (Reino Unido) }\end{array}$} & 0 & 0 & 0 & 7 & 2 & 1 & 10 \\
\hline &, $0 \%$ &, $0 \%$ &, $0 \%$ & $1,9 \%$ &, $5 \%$ &, $3 \%$ & $2,7 \%$ \\
\hline
\end{tabular}




\begin{tabular}{|c|c|c|c|c|c|c|c|}
\hline \multirow{2}{*}{ ENTV (Argelia) } & 0 & 1 & 2 & 41 & 1 & 3 & 48 \\
\hline &, $0 \%$ &, $3 \%$ &, $5 \%$ & $11,1 \%$ &, $3 \%$ &, $8 \%$ & $12,9 \%$ \\
\hline \multirow{2}{*}{ ERTU (Egipto) } & 0 & 0 & 0 & 93 & 2 & 0 & 95 \\
\hline &, $0 \%$ &, $0 \%$ &, $0 \%$ & $25,1 \%$ &, $5 \%$ &, $0 \%$ & $25,6 \%$ \\
\hline \multirow{2}{*}{$\begin{array}{l}\text { France 2 } \\
\text { (Francia) }\end{array}$} & 0 & 0 & 0 & 19 & 0 & 0 & 19 \\
\hline &, $0 \%$ &, $0 \%$ &, $0 \%$ & $5,1 \%$ &, $0 \%$ &, $0 \%$ & $5,1 \%$ \\
\hline \multirow{2}{*}{$\begin{array}{l}\text { RAI 1 } \\
\text { (Italia) }\end{array}$} & 2 & 0 & 0 & 55 & 1 & 0 & 58 \\
\hline &, $5 \%$ &, $0 \%$ &, $0 \%$ & $14,8 \%$ & $3 \%$ & , $0 \%$ & $15,6 \%$ \\
\hline \multirow{2}{*}{$\begin{array}{l}\text { SNRT } \\
\text { Al Aoula } \\
\text { (Marruecos) }\end{array}$} & 4 & 0 & 1 & 27 & 4 & 0 & 36 \\
\hline & $1,1 \%$ &, $0 \%$ &, $3 \%$ & $7,3 \%$ & $1,1 \%$ &, $0 \%$ & $9,7 \%$ \\
\hline \multirow{2}{*}{$\begin{array}{l}\text { Syria Channel } \\
\text { (Siria) }\end{array}$} & 0 & 1 & 1 & 65 & 0 & 2 & 69 \\
\hline & , $0 \%$ &, $3 \%$ &, $3 \%$ & $17,5 \%$ &, $0 \%$ &, $5 \%$ & $18,6 \%$ \\
\hline \multirow{2}{*}{ TVE 1 (España) } & 2 & 0 & 0 & 32 & 2 & 0 & 36 \\
\hline &, $5 \%$ &, $0 \%$ &, $0 \%$ & $8,6 \%$ &, $5 \%$ &, $0 \%$ & $9,7 \%$ \\
\hline \multirow{2}{*}{ TOTAL } & 8 & 2 & 4 & 339 & 12 & 6 & 371 \\
\hline & $2,2 \%$ &, $5 \%$ & $1,1 \%$ & $91,4 \%$ & $3,2 \%$ & $1,6 \%$ & $100,0 \%$ \\
\hline
\end{tabular}

Fuente: Elaboración propia

Los aspectos relacionados con Religión aparecen solamente en el 7,3\% del total de las unidades de análisis a través de: relaciones intrarreligiosas $(3,2 \%)$, relación religión-Estado $(1,6 \%)$, diálogo y conocimiento mutuo $(1,3 \%)$, rasgos específicos $(0,5 \%)$, discriminación religiosa $(0,3 \%)$ y relaciones interreligiosas $(0,3 \%)$.

En ERTU, Syria Channel y TVE 1 únicamente hallamos informaciones que hagan referencia a las relaciones intrarreligiosas, representando el $0,3 \%$, el $0,5 \%$ y $0,3 \%$, respectivamente, del total las piezas estudiadas.

En RAI 1 localizamos dos subtemas: relación religión-Estado $(1,3 \%)$ y relaciones intrarreligiosas $(0,3 \%)$.

En ENTV Argelia se observa la presencia informaciones que incluyan las relaciones intrarreligiosas $(0,5 \%)$, diálogo y conocimiento muto $(0,3 \%)$ y relaciones interreligiosas $(0,3 \%)$.

La marroquí SNRT 1 Al Aoula incorpora los subtemas siguientes: diálogo y conocimiento mutuo $(1,1 \%)$, relaciones intrareligiosas $(0,5 \%)$, rasgos específicos $(0,3 \%)$ y relación religión-Estado $(0,3 \%)$.

En France 2 observamos la presencia de noticias con referencia a las relaciones intrarreligiosas $(0,8 \%)$ y a rasgos específicos $(0,3 \%)$.

La BBC One solamente alude a la religión a través de la discriminación religiosa, con un $0,3 \%$.

\begin{tabular}{|c|c|c|c|c|c|c|c|c|}
\hline \multirow[b]{2}{*}{ TV } & \multicolumn{7}{|c|}{ Tabla 5. Religión } & \multirow[b]{2}{*}{ Total } \\
\hline & $\begin{array}{c}\text { Diálogo y } \\
\text { conocimiento } \\
\text { mutuo }\end{array}$ & $\begin{array}{l}\text { Discriminación } \\
\text { religiosa }\end{array}$ & No hay & $\begin{array}{c}\text { Rasgos } \\
\text { específicos }\end{array}$ & $\begin{array}{l}\text { Relación } \\
\text { religión - } \\
\text { Estado }\end{array}$ & $\begin{array}{c}\text { Relaciones } \\
\text { interreligiosas }\end{array}$ & $\begin{array}{l}\text { Relaciones intra- } \\
\text { religiosas }\end{array}$ & \\
\hline \multirow{2}{*}{$\begin{array}{l}\text { BBC One } \\
\text { (Reino Unido) }\end{array}$} & 0 & 1 & 9 & 0 & 0 & 0 & 0 & 10 \\
\hline &, $0 \%$ &, $3 \%$ & $2,4 \%$ &, $0 \%$ &, $0 \%$ &, $0 \%$ &, $0 \%$ & $2,7 \%$ \\
\hline \multirow{2}{*}{ ENTV (Argelia) } & 1 & 0 & 44 & 0 & 0 & 1 & 2 & 48 \\
\hline &, $3 \%$ &, $0 \%$ & $11,9 \%$ &, $0 \%$ &, $0 \%$ &, $3 \%$ &, $5 \%$ & $12,9 \%$ \\
\hline \multirow{2}{*}{ ERTU (Egipto) } & 0 & 0 & 94 & 0 & 0 & 0 & 1 & 95 \\
\hline &, $0 \%$ &, $0 \%$ & $25,3 \%$ &, $0 \%$ &, $0 \%$ &, $0 \%$ &, $3 \%$ & $25,6 \%$ \\
\hline
\end{tabular}




\begin{tabular}{|c|c|c|c|c|c|c|c|c|}
\hline \multirow{2}{*}{$\begin{array}{l}\text { France 2 } \\
\text { (Francia) }\end{array}$} & 0 & 0 & 15 & 1 & 0 & 0 & 3 & 19 \\
\hline &, $0 \%$ &, $0 \%$ & $4,0 \%$ & $3 \%$ &, $0 \%$ &, $0 \%$ & $8 \%$ & $5,1 \%$ \\
\hline \multirow{2}{*}{$\begin{array}{l}\text { RAI 1 } \\
\text { (Italia) }\end{array}$} & 0 & 0 & 52 & 0 & 5 & 0 & 1 & 58 \\
\hline &, $0 \%$ &, $0 \%$ & $14,0 \%$ &, $0 \%$ & $1,3 \%$ &, $0 \%$ &, $3 \%$ & $15,6 \%$ \\
\hline \multirow{2}{*}{$\begin{array}{l}\text { SNRT } \\
\text { Al Aoula } \\
\text { (Marruecos) }\end{array}$} & 4 & 0 & 28 & 1 & 1 & 0 & 2 & 36 \\
\hline & $1,1 \%$ &, $0 \%$ & $7,5 \%$ &, $3 \%$ &, $3 \%$ &, $0 \%$ &, $5 \%$ & $9,7 \%$ \\
\hline \multirow{2}{*}{$\begin{array}{l}\text { Syria Channel } \\
\text { (Siria) }\end{array}$} & 0 & 0 & 67 & 0 & 0 & 0 & 2 & 69 \\
\hline &, $0 \%$ &, $0 \%$ & $18,1 \%$ &, $0 \%$ &, $0 \%$ &, $0 \%$ &, $5 \%$ & $18,6 \%$ \\
\hline \multirow{2}{*}{ TVE 1 (España) } & 0 & 0 & 35 & 0 & 0 & 0 & 1 & 36 \\
\hline &, $0 \%$ &, $0 \%$ & $9,4 \%$ &, $0 \%$ &, $0 \%$ &, $0 \%$ &, $3 \%$ & $9,7 \%$ \\
\hline \multirow{2}{*}{ TOTAL } & 5 & 1 & 344 & 2 & 6 & 1 & 12 & 371 \\
\hline & $1,3 \%$ & $3 \%$ & $92,7 \%$ &, $5 \%$ & $1,6 \%$ &, $3 \%$ & $3,2 \%$ & $100,0 \%$ \\
\hline
\end{tabular}

Fuente: Elaboración propia

\section{Conclusiones}

La zona euromediterránea es un área para considerar con interés en el análisis de los espacios comunicativos en los próximos años. Del análisis de las informaciones ofrecidas en los principales telenoticias de las televisiones de la región hay que tener muy en cuenta los enfoques que han planteado y que ponen de manifiesto las tendencias informativas. Asimismo, cobra especial relevancia el papel de las emisoras de televisión gestionadas directamente por los gobiernos que deberían cumplir su misión de servicio público, contribuyendo a los procesos de diálogo e integración entre los países de la zona.

Las informaciones que tienen un enfoque dirigido a contar las actuaciones políticas son las que marcan la agenda en el espacio euromediterráneo. De todos modos la economía, seguida de lo social y el militar y/o bélico son los otros principales enfoques que se observan del análisis de contenido aplicado a las televisiones más relevantes de este ámbito. Otros enfoques como el cultural o el religioso son minoritarios en la muestra considerada.

Considerando los temas presentes en los contenidos, la política ocupa un lugar destacado. Más de un $70 \%$ de las noticias analizadas trataron aspectos relacionados con política y legislación, siendo el tema principal las políticas multilaterales o bilaterales, seguido de derecho internacional y derechos humanos y de partidos políticos.

El asunto predominante cuando analizamos las noticias relativas a economía es la crisis económica mundial, seguido de recursos naturales y medioambiente aunque a una distancia más que notable.

De los cuatro temas considerados en el presente estudio, la cultura y la religión ocupan un espacio secundario (menos de un 10\%) en los telediarios analizados. La cultura aparece esencialmente a través de contenidos sobre patrimonio cultural o diálogo y conocimiento mutuo. No obstante, las relaciones interculturales, un elemento clave para la región, se trataron en poco más del 1\% de la información total analizada. En cuanto a la religión, las relaciones intrarreligiosas son las que aparecen de forma mayoritaria seguido de relación religión-Estado, mientras las relaciones entre diferentes religiones fueron tratadas en apenas una de las 371 noticias analizadas. 
A partir de los datos constatados, se verifica que las televisiones públicas de los países mediterráneos no dedican el mismo espacio en sus telediarios a los diferentes ámbitos de acción destacados por las iniciativas e instancias supranacionales. Los medios de comunicación tienen un papel fundamental en estos procesos de acercamiento e integración regional, por lo que las televisiones, en especial las de titularidad pública, deberían dedicar más atención y protagonismo a todos los aspectos relevantes para la consolidación del espacio euromediterráneo.

\section{Referencias bibliográficas}

BARDIN, Laurence (1986): Análisis de contenido. Barcelona, AKAL.

BARDIN, Laurence (1995): Análise de conteúdo. Lisboa, Edições 70.

BERELSON, Bernad (1986): Análisis de contenido. Madrid, Akal/Universitaria.

BERGUER, Peter L. y LUCKMAN, Thomas (1976): La construcción social de la realidad. Buenos Aires, Amorrortu.

HOLSTI, Ole R. (1968): “Content analysis”, en LINDZEY, Gardner y ARONSON, Elliot: The handbook of social Psychology. Vol. 2. Research Methods, AddisonWesley, Reading, Mass.

KRIPPENDORFF, Klaus (1990): Metodología del análisis de contenido. Teoría y práctica. Barcelona, Paidós Comunicación.

RIZO, Marta (2009): "Sociología fenomenológica y comunicología: Sociología Fenomenológica y sus aportes a la comunicación interpersonal y mediática". Revista Fronteiras -Estudos Midiáticos, no 11 (1): 25-32, janeiro/abril 2009.

VELÁZQUEZ, Teresa (1992): Los políticos y la televisión. Aportaciones de la teoría del discurso al diálogo televisivo. Barcelona, Ariel.

VELÁZQUEZ, Teresa (2011): "Las técnicas cualitativas del análisis socio-semiótico" en: VILCHES, Lorenzo (coord.): La investigación en comunicación. Métodos y técnicas en la era digital. Barcelona, Gedisa, cap. 8: 237-265. 